\title{
The quasi-periodicity of the Minority Game revisited
}

\author{
Gabriel Acosta $^{\mathrm{a}}$, Inés Caridi ${ }^{\mathrm{a}, *}$, Sebastián Guala ${ }^{\mathrm{b}}$, Javier Marenco ${ }^{\mathrm{b}}$ \\ ${ }^{a}$ Facultad de Ciencias Exactas y Naturales, Universidad de Buenos Aires, \\ Pabellón I, Ciudad Universitaria, (1428) Buenos Aires, Argentina \\ ${ }^{b}$ Instituto de Ciencias, UNGS, \\ J. M. Gutiérrez 1150, (1613) Los Polvorines, Argentina
}

\begin{abstract}
We analyze two well-known related aspects regarding the sequence of minority sides from the Minority Game $(M G)$ in its symmetric phase: period-two dynamics and quasi-periodic behavior. We also study the sequence of minority sides in a general way within a graph-theoretical framework. In order to analyse the outcome dynamics of the $\mathrm{MG}$, it is useful to define the $M G^{\text {prior }}$, namely an $M G$ with a new choosing rule of the strategy to play, which takes into account both prior preferences and game information. In this way, each time an agent is undecided because two of her best strategies predict different choices while being equally successful so far, she selects her a priori favorite strategy to play, instead of performing a random tie-break as in the $M G$. This new choosing rule leaves the generic behavior of the model unaffected and simplifies the game analysis. Furthermore, interesting properties arise which are only partially present in the $M G$, like the quasi-periodic behavior of the sequence of minority sides, which turns out to be periodic for the $M G^{\text {prior }}$.
\end{abstract}

Keywords: Minority game; quasi-periodic behavior; choosing rule PACS: 89.65.Gh, 82.20.Wt

\section{Introduction}

The Minority Game $(M G)$ [1] is an agent-based model inspired in real complex systems which presents interesting collective properties like coordi-

\footnotetext{
*Corresponding author

Email address: ines@df.uba.ar (Inés Caridi)
} 
nation among agents $[2,3,4]$. In the original formulation of the game, $N$ agents (usually odd) must simultaneously choose one out of two alternatives: 0 or 1 , and the winners are those who happen to be in the minority group. The $M G$ tries to capture essential characteristics of some real situations in which belonging to the minority group turns out to be the most convenient situation (as, e.g., financial systems, traffic problems, and data networks).

At each step $t$ of the game, $N_{0}(t)$ agents choose side 0 and $N_{1}(t)$ agents choose side 1 , so that $N_{0}(t)+N_{1}(t)=N$. The system state $\mu \in\{0,1\}^{m}$ is the only global information available for agents to make decisions. After each step, the state $\mu$ is updated on the basis of a certain updating rule. For example, in the original MG, $\mu$ is determined by the sequence of minority sides in the last $m$ steps of the game.

A strategy is a function that assigns a prediction ( 0 or 1$)$ to each possible state. In our case, every agent has $s=2$ strategies at hand and at each step of the game, she plays using her best one. Whenever an agent's two strategies are equally ranked, she chooses one of them randomly.

An instance $I$ of the $M G$ with $s=2$ is a particular assignment of two strategies to the agents, $I=\left\{\left(e_{1}^{1}, e_{2}^{1}\right),\left(e_{1}^{2}, e_{2}^{2}\right), \cdots\right\}$. For $i=1, \ldots, N$, the pair $\left(e_{1}^{i}, e_{2}^{i}\right)$ represents the set of strategies assigned to the agent $i$. We define a realization $\mathcal{E}$ of the game as a pair $\mathcal{E}=\left\{\mathcal{S}_{\mathcal{E}}, I\right\}$, where $\mathcal{S}_{\mathcal{E}}=\left\{\tilde{\mu}^{1}, \tilde{\mu}^{2}, \cdots\right\}$ is a sequence of states (generated by any updating rule), and $I$ is an instance of the $M G$.

The most studied variable in the $M G$ is the reduced variance $\sigma^{2} / N=$ $\left\langle\left(N_{1}-N / 2\right)^{2} / N\right\rangle_{\mathcal{E}}[5]$. It measures the population's waste of resources by averaging -over time and over different realizations $\mathcal{E}$ - the quadratic deviation from $N / 2$ of the number of agents that choose a fixed side (for example, $N_{1}$ ). When crowds emerge in the game, their contribution to $\sigma^{2} / N$ is very important, indicating that fewer resources are being allocated to the population as a whole. On the other hand, for certain values of the parameters $m, N$, and $s, \sigma^{2} / N$ results smaller than that obtained for a game in which each of the $N$ agents randomly chooses between the two sides.

Period Two Dynamics (PTD) in the sequence of the minority sides was first observed by Savit et al. [9] within the symmetric phase of the MG. The PTD can be summarized in the following way: if a state $\mu$ appears at step $t$ and this appearence is odd (i.e., the first, third, etc. step in which state $\mu$ occurs), then in the next (and hence even) appearance of $\mu$, the outcome of the game is very likely to be the opposite of that obtained in the step $t$. Broadly speaking, this dynamics is due to the fact that on even occurrences 
of $\mu$, crowds of agents will move together to the side rewarded in the previous odd appearance of the same state [9]. When PTD is met with probability 1 (i.e., when $P_{P T D}=1$ ), we call it Strict Period Two Dynamics (SPTD).

Acosta et al. have analitically solved the Full Strategy Minority Game $(F S M G)[14,10]$, a maximal instance of the $M G$, which includes a single copy of every potential agent. For example, in a game with parameters $m$ and $s=2$, the number of potential agents is $\mathcal{N}=\left(\begin{array}{l}\mathcal{L} \\ 2\end{array}\right)+\mathcal{L}$, where the first term represents all agents with two different strategies and the second term represents the number of agents whose two strategies are identical. Thus, the number of agents of the FSMG is a function of $m$. Certain strategies' symmetries, broken in the $M G$, can be fully exploited in the FSMG. This approach leads us to show that the FSMG verifies the SPTD for even occurrences of the states. The advantage of this approach lies in considering the $M G$ as a statistical sample of size $N$ of the FSMG. As a consequence, theoretical results for the FSMG can be used to compute approximated values of the key variable $\sigma^{2} / N$ for the standard $M G$ in the symmetric phase, as well as for other versions of the $M G$ based on different updating rules, like the random updating rule introduced in [6] $\left(M G_{\text {rand }}\right)$ and the periodic updating rule introduced in [13] (denoted by $M G_{p e r}$ ).

It is important to note that by an even (resp. odd) occurrence of a state we refer to the situation in which the current state $\mu_{p}$ at step $t$ has appeared an odd (resp. even) number of times up to the step $t-1$. Since $\mu_{p}$ appears again at step $t$, we consider this appearence to be an even (resp. odd) occurrence. This is an important remark in order to understand the results in [14, 10] and the appendix of this paper.

In this work, we first show that PTD is also met for odd occurrences of the states in the symmetric phase, but this is not accompanied by a crowd effect. This dynamics is reported as the antipersistence of the attendance. Jefferies et al. [11] show the MG behaves as a stochastically-disturbed deterministic system due to the random rule to resolve situations of tied strategies, by averaging over this stochasticity in order to get a deterministic dynamics of the MG. They also show that the trajectory of the outcomes of the deterministic MG on a de Bruijn graph is periodic within an Eulerian trail. Our article is closely related with that of Jefferies et al. Indeed, we use the FSMG framework instead of the RSS (Reduced Strategy Space), and we take into account the effect of undecided agents, which in fact not change the underlying dynamics. We also give elementary proofs for the connection among the periodicity of the MG outcomes, the eulerian path on the de 
Bruijn graph, and the antipersistence of the attendance (or the PTD). On the other hand, the works of Zheng et al. [12] and Liaw et al. [13] show the existence of quasiperiods in the sequence of minority sides in the $M G$. In fact, the outcome of minority sides resulting from a game with $m=2$, we can observe that there are two particular sequences of size 8 which appear several times in the sequence (though both sequences do not necessarily appear in the same realization of the game). These facts encourage us to propose an alternative choosing rule of the strategy to play, for which each agent has an a priori favorite strategy to use in case of indecision. We call $M G^{\text {prior }}$ the resulting model. This modification holds the same behavior, which moreover facilitates an analytical understanding of the general PTD observed in the simulations of the $M G$. Furthermore, interesting properties arise which are only partially present in the $M G$, like the quasi-periodic behavior of the sequence of minority sides, which turns out to be periodic for the $M G^{\text {prior }}$.

Just like in the $M G$, we can also define the Full Strategy Minority Game with the prior choosing rule of strategies $\left(F S M G^{\text {prior }}\right)$ as the maximal instance of the $M G^{\text {prior }}$ which verifies that $I$ is the complete set of potential agents of the $M G^{\text {prior }}$. In Appendix A we prove that the $F S M G^{\text {prior }}$ necessarily verifies the SPTD for even occurrences of the states. Finally, we prove in Appendix B the equivalence between the $M G^{\text {prior }}$ and the $M G_{\text {rand }}^{\text {prior }}$ (i.e., $M G^{\text {prior }}$ with random updating rule by following [6]) in terms of $\sigma^{2} / N$.

\subsection{Additional definitions}

We include here some specific definitions, notations and results about the $M G$ model. The number of states of the sytem is $\mathcal{H}=2^{m}$, and we denote by $S_{\mathcal{H}}$ the complete set of states. The number of strategies is $\mathcal{L}=2^{\mathcal{H}}$, and the complete set of strategies $S_{\mathcal{L}}$ is known as the Full Strategy Space. At each step of the game, each agent plays using her best strategy (i.e., the one which has predicted the minority side the greatest number of times). To this end, the strategies of every agent are ranked according to the number of rounds that each one has correctly predicted the minority side. If the strategies are tied then in the $M G$ the agent selects one of her strategies at random, whereas in the proposed $M G^{\text {prior }}$ the agent selects her favorite strategy.

The behavior of the MG as a function of the parameters has been characterized by two phases: a symmetric one and an asymmetric one. In the curve of $\sigma^{2} / N$ vs $\alpha=2^{m} / N$, the minimun value of $\sigma^{2} / N$ attained in $\alpha=\alpha_{c}$ is associated with the broken symmetry. When $\alpha<\alpha_{c}$ the MG is in the symmetric phase, and when $\alpha>\alpha_{c}$ the MG is in the asymmetric phase. Soon 
after the $M G$ was introduced by Challet and Zhang [1], Cavagna proposed a new updating rule for the state of the system [6] (here denoted $M G_{\text {rand }}$ ) for which the state is established at random (from a uniform distribution) in each step. This modification essentially gives rise to a model bearing the same qualitative behavior $[7,8]$.

\section{Period Two and Periodic/Quasiperiodic Dynamics of the $M G^{\text {prior }}$ and MG by simulations}

In this section we address the behavior of $\sigma^{2} / N$, the PTD, and the periodicity properties of the $M G$ and the $M G^{\text {prior }}$. The only difference between $M G$ and $M G^{\text {prior }}$ consists in that ties are broken a priori during the assignment of strategies to the players. Thus, an instance $(I)$ of the $M G^{\text {prior }}$ is a particular ordered assignment of strategies to the agents, $I=\left\{\left(e_{1}^{1}, e_{2}^{1}\right),\left(e_{1}^{2}, e_{2}^{2}\right), \cdots\right\}$. For agent $i$, the order of the pair $\left(e_{1}^{i}, e_{2}^{i}\right)$ defines her preferences, so that $e_{1}^{i}$ is defined to be her favorite strategy when $e_{1}^{i}$ and $e_{2}^{i}$ are tied in virtual points (the points assigned to strategies in order to record their success in the game).

Numerical simulations of the $M G^{\text {prior }}$ evidence that $\sigma^{2} / N$ shows the same behavior as in the $M G$ case. The only difference appears in the fluctuations of the reduced variance of the $M G^{\text {prior }}$, which are bigger than in the $M G$ case, as shown in Figure 1. Since the $M G^{\text {prior }}$ reproduces similar dynamics to that of the $M G$, we consider the $M G^{\text {prior }}$ to be of interest for us, despite the fact that this model shows a completely deterministic development. This fact allows a potentially easier analysis regarding the sequence of minority sides and the PTD both for even and odd occurrences of the states.

Taking into account the sequence of the minority sides resulting from the simulation of the $M G$, it is possible to compute the probability that the PTD is met in a given realization of the game. Actually, we have computed the probability for PTD to take place $\left(P_{P T D}\right)$ as 1 minus the probability of breaking the PTD for the first time in a given simulation of the game, as it was computed in [10]. Moreover, this can be done by distinguishing the calculation for the even and odd cases of occurrences of the states. These results are shown in Figure 2 and reflect that if $2^{m} / N \ll 1$ then $P_{P T D} \sim 1$ both for even and odd occurrences of the states of the $M G^{\text {prior }}$, while for the $M G$ case we have $P_{P T D} \sim 1$ for even occurrences and $P_{P T D} \sim 0.8$ for odd occurrences. In the latter, however, there are no crowd effects, as Figure 3 


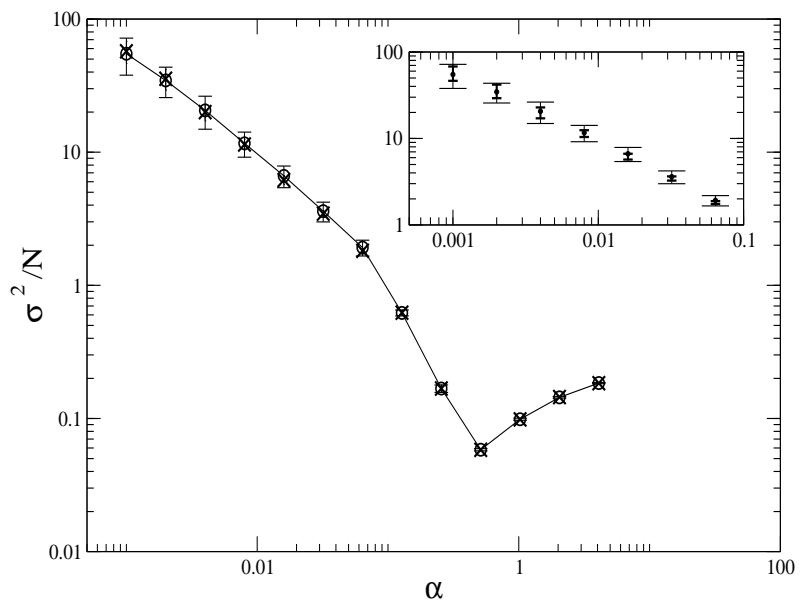

Figure 1: The X symbols show $\sigma^{2} / N$ as a function of $\alpha$ for the $M G$ for different values of $m$ (from 2 to 14) and $N=4001$, and the circular symbols contain the same measurements for the $M G^{\text {prior }}$. For each value of $m, 100$ runs have been performed, each one of $T=100000$ time steps discarding the first 50000 steps. In the inset, error bars of $\sigma^{2} / N$ are shown for $m=2, \ldots, 8$. The widest error bars correspond to the $M G^{\text {prior }}$.

shows. In this figure we plot the deviations of $\sigma_{e}^{2} / N$ and $\sigma_{o}^{2} / N$ for even and odd occurrences of the states, respectively. High values of $\sigma_{e}^{2} / N$ evidence a crowd effect. On the other hand, $\sigma_{o}^{2} / N \sim 0.25$ reflects that fluctuacions of $N_{1}$ with respect to the mean value are like the case in which agents randomly choose their decision, without any type of crowd effect.

The fact that $M G^{\text {prior }}$ model meets SPTD for both even and odd occurrences of the states when $2^{m} / N \ll 1$ is related with the periodicity of the game for these values of $m$ and $N$. We shall return to this topic in Section 3.

Let us consider a periodic sequence of minority sides for a game with $m=$ 2 satisfying the SPTD (i.e., meeting PTD for all steps, involving both even and odd occurrences of every state). There are only two possible sequences with these characteristics, namely 00011101 and 11100010 . In fact, these two sequences correspond to the same case by swapping sides. It is important to note that these sequences have length $2 \mathcal{H}=8$ (later in this work we will show that there are only two possible sequences for $m=2$ meeting SPTD and periodicity, and why the corresponding period is $2 \mathcal{H}=8$ ).

A directed graph is a pair $G=(V, E)$, where $V$ is a finite set of so-called nodes and $E \subseteq\{(i, j): i, j \in V, i \neq j\}$ is the set of arcs or directed edges. In order to make the periodicity behavior of $M G^{\text {prior }}$ and the quasi-periodicity 

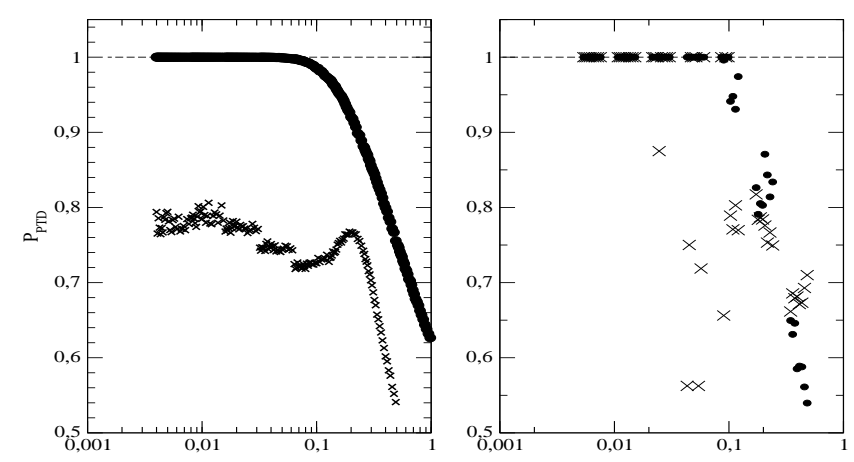

Figure 2: Probability $P_{P T D}$ for PTD to take place in even (circles) and odd (X) occurrences of the states as a function of $\alpha$, calculated as one minus the probability of breaking the PTD for the first time in a given simulation of the game. In the left, simulations for the $M G$ model; in the right, simulations for the $M G^{\text {prior }}$ model. In the simulations we have computed $P_{P T D}$ as follows: at each even occurrence of each state, if after the poll of players the PTD is not fulfilled (i.e., the minority side agrees with that obtained after the previous odd occurrence of the same state), then we consider that this step does not contribute to $P_{P T D}$. At the same time, present state and virtual points are assigned as if the PTD had not failed. This way we compute the probability of breaking the PTD for the first time in the game.

behavior of $M G$ evident, we shall represent the outcomes of a particular game through a directed graph. To this end, arrange the set of minority sides into chains of length $2 \mathcal{H}(=8$ for $m=2)$. At each game step, the new minority side is used to construct the new chain, along with the previous $2 \mathcal{H}-1$ minority sides, by attaching this bit at the end. Each node of the graph represents a particular chain of minority sides. For example, in Figure 4.a the outcomes of a realization of the $M G^{\text {prior }}$ only discarding a few beginning steps are represented. Since the outcomes lie in the periodic sequence ...0001110100011101... then only eight nodes appear in the graph, namely 00011101, 00111010, 01110100, 11101000, 111010001, 10100011, 01000111, 10001110. A link between node $i$ and $j$ is established every time the game goes from node $i$ to node $j$. The node sizes are proportional to the probability of occurrence of each node. Figure 4.b corresponds to another realization of the $M G^{\text {prior }}$ model, which results in the periodic sequence 11100010. In both figures, all the links of the graph are equally toured, as shown in the 


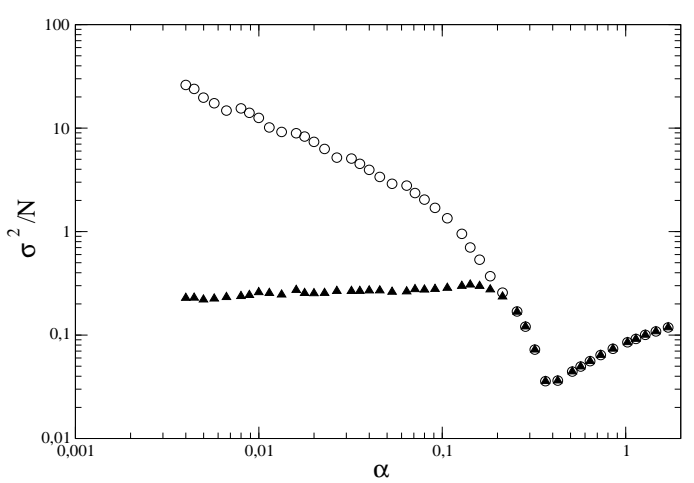

Figure 3: Values of $\sigma_{e}^{2} / N$ (circles) and $\sigma_{o}^{2} / N$ (triangles) as a function of $\alpha$ for the $M G$ for different values of $m$ (in the range from 2 to 14) and $N$. For each value of $N$ and $m$ we perform 100 runs, each one of $T=100000$ time steps discarding the first 50000 steps for values of $m<8$; and each one of $T=500000$ time steps discarding the first 250000 steps for values of $m \geq 8$.

frequencies of occurrence of each link in Tables 1 and 2. The node sizes are equal, and only the eight nodes associated with a periodic sequence are present. These two facts are in accordance with the periodicity of the game (periodicity reached after less than 20 steps for these particular realizations). Every realization of the $M G^{\text {prior }}$ model which meets SPTD for even and odd occurences of the states results in one of these two possible graphs: either the graph associated with the sequence 00011101 or the graph associated with the sequence 11100010. The periodic sequence that the system chooses is determined by the initial conditions: the initial state and the instance $I$ of the realization.

In Figure 5 we show simulations of the $M G^{\text {prior }}$ for $m=2,3,4,5$ and $N=$ 1001, where the periodicity of the sequence of $N_{1}$ is reflected. Furthermore, Figure 6 illustrates the strong periodicity of the $M G^{\text {prior }}$ for $m=2$ and $N=101$ in the form of a "2D code", where each pixel represents an agent who can choose either 1 (black) or 0 (white). These results show that not only the sequence of outcomes is periodic but that the behavior of each agent is periodic too, accumulating more evidence concerning the periodicity of the $M G^{\text {prior }}$. The same behavior is observed for $m=2$ and $N=1001$, but in this case the 1001 pixels are more difficult to appreciate in a small graph. 


\begin{tabular}{|c|c|c|}
\hline Source node & Destination node & Frequency \\
\hline 10100011 & 01000111 & 248 \\
01000111 & 10001110 & 248 \\
10001110 & 00011101 & 248 \\
00011101 & 00111010 & 248 \\
00111010 & 01110100 & 248 \\
01110100 & 11101000 & 248 \\
11101000 & 11010001 & 248 \\
11010001 & 10100011 & 249 \\
\hline
\end{tabular}

Table 1: Frequencies of occurrence of the links in the graph of Figure 4.a. Data correspond to a realization of the $M G^{\text {prior }}$ with $N=1001, m=2$, and 2000 time steps, discarding the first 15 steps.

Different periodic sequences exist, e.g., with period length $L=16$ and $L=24$, although in these cases the SPTD is not met for odd occurrences of the states. We performed 500 realizations of the $M G^{\text {prior }}$ with $N=101$ and $m=2$, and we found 15 realizations in which the period was greater than $L=8$. In 13 such realizations the period was $L=16$ and in the 2 remaining cases the period was $L=24$. We show some of these results in Table 3. In these simulations we have $\alpha \sim 0.04$ in order to ensure that the $M G^{\text {prior }}$ is in the region in which SPTD is met for even occurrences, but the SPTD may be broken in odd occurrences of the states.

Figures 4.c and 4.d show the graphs associated with the $M G$ for two further realizations. The nodes appearing with a probability smaller than $0.6 \%$ have been deleted from Figure 4.c, and nodes with probability smaller than $0.9 \%$ have been deleted from Figure 4.d. In the case of Figure 4.d, the two sets of nodes consistent with the two periodic sequences 11100010 and 00011101 are present, but there are also other nodes in the graph with smaller associated frequencies. We call outer path to a path joining two nodes from the cycle. Note that the two outer paths in Figure 4.d have the same size and opposite directions (the first one goes from the cycle depicted in Figure 4.a to the cycle in Figure 4.b, whereas the other outer path goes the other way around). We shall further discuss these observations in the last section. This fact reflects that the system moves from one cycle to the other cycle, usually one of them having a greater frequency of occurrence.

In the case of Figure 4.c, only one of the periodic cycles is depicted (the 


\begin{tabular}{|c|c|c|}
\hline Source node & Destination node & Frequency \\
\hline 11100010 & 11000101 & 248 \\
11000101 & 10001011 & 249 \\
10001011 & 00010111 & 249 \\
00010111 & 00101110 & 249 \\
00101110 & 01011100 & 249 \\
01011100 & 10111000 & 249 \\
10111000 & 01110001 & 249 \\
01110001 & 11100010 & 249 \\
\hline
\end{tabular}

Table 2: Frequencies of occurrence of the links in the graph of Figure 4.a. Data correspond to a realization of the $M G^{\text {prior }}$ with $N=1001, m=2$, and 2000 time steps, discarding the first 9 steps.

\begin{tabular}{|c|c|c|}
\hline $\begin{array}{c}P_{P T D} \text { in } \\
\text { odd states }\end{array}$ & $\begin{array}{c}\text { Periodic } \\
\text { sequence }\end{array}$ & $\begin{array}{c}\text { Period } \\
\text { length } L\end{array}$ \\
\hline 0.5 & 1110010111010000 & 16 \\
0.5 & 0000111101001101 & 16 \\
0.25 & 0000101111001101 & 16 \\
0.25 & 0001000110111101 & 16 \\
0.25 & 1110010000101110 & 16 \\
0.5 & 1101001110000101 & 16 \\
0.5 & 0000110100111101 & 16 \\
0.5 & 1010011101110000 & 16 \\
0.25 & 1111000101011000 & 16 \\
0.5 & 0111010111000010 & 16 \\
0.5 & 0111001000011101 & 16 \\
0.5 & 1000011101011100 & 16 \\
0.25 & 1111010110000100 & 16 \\
0.5 & 111101000011101011001000 & 24 \\
0.5 & 000011010011101111000101 & 24 \\
\hline
\end{tabular}

Table 3: Periodic sequences of length $L>8$ appear in realizations of the $M G^{\text {prior }}$ with $N=101$ and $m=2$. We performed 500 realizations and only 15 of them resulted in periodic sequences both with period length $L>8$ and with $P_{P T D}<1$ in odd occurrences of the states, but $P_{P T D}=1$ in even occurrences of the states. 

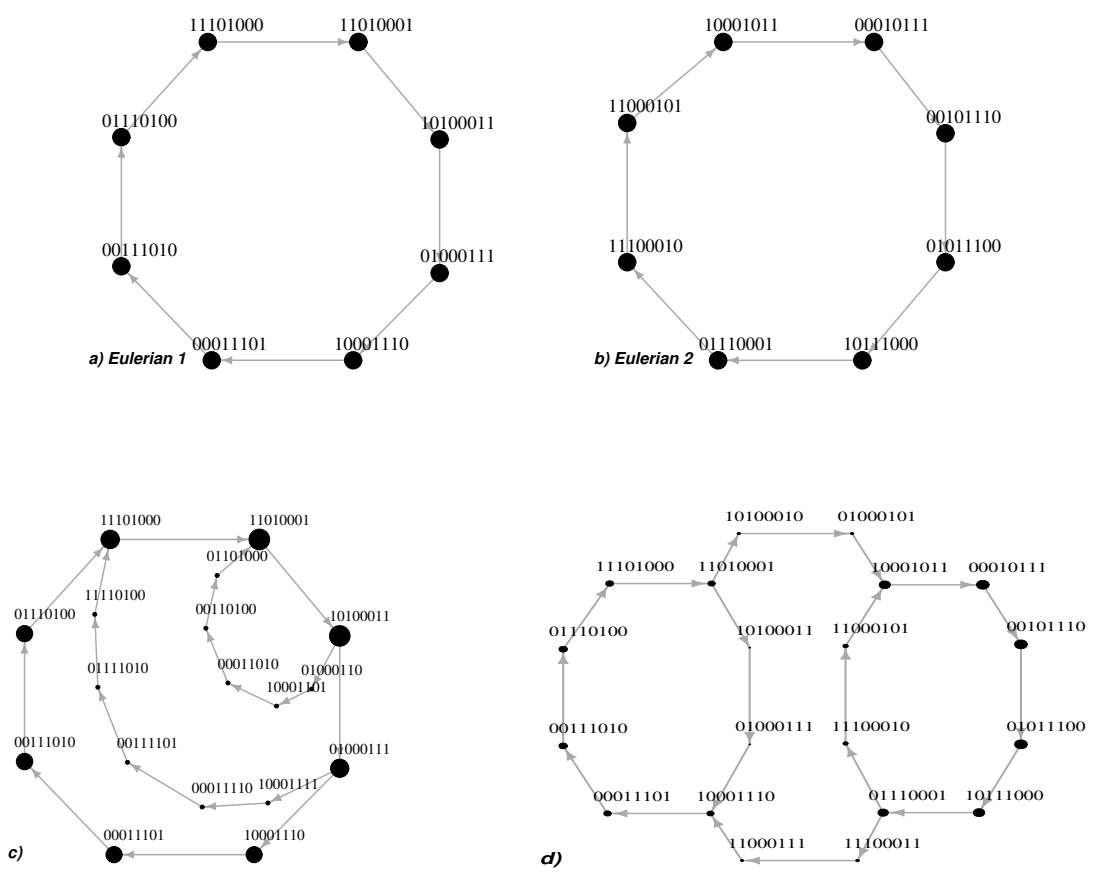

Figure 4: Figures a and b show the cycles in an $M G^{\text {prior }}$ realization (in Section IV we will prove that they correspond to eulerian paths of a De Bruijn graph of order $m$ ). Figures c and d correspond to two realizations of the $M G$. In all cases there are $N=1001$ agents, we set $m=2$, and we perform 2000 game steps in the $M G$ cases. Graphs made using the igraph package of $\mathrm{R}[15,16]$.

other cycle appears in the simulation but its frequency of occurrence is too small and hence below the threshold for inclusion in the figure). The fact that other links and nodes are present (with smaller associated frequency of occurrence) reflects the quasi-periodic behavior of the $M G$.

For $m>2$ there are more than two possible sequences that meet SPTD for all the steps. In section 3 we will return to this general case.

\section{FSMG ${ }^{\text {prior }}$ : definition and properties}

We define the Full Strategy Minority Game with an a priori favorite strategy $\left(F S M G^{\text {prior }}\right)$ to be an instance of the $M G^{\text {prior }}$ which includes a 
single copy of every potential agent of this game.

As mentioned in the introduction, $\mathcal{H}=2^{m}$ and $\mathcal{L}=2^{\mathcal{H}}$ are the number of different states and different strategies for the $M G$ and $M G^{\text {prior }}$ with parameter $m$, respectively. Then, $F S M G^{\text {prior }}$ has $\mathcal{N}=\mathcal{L}^{2}$ different agents, because there are $\mathcal{N}$ ways of choosing an ordered pair of strategies from the Full Strategy Space.

In the Appendix A we show that the FSMG ${ }^{\text {prior }}$ necessarily meets the SPTD for any realization $\mathcal{E}$.

In the following we will prove that a minority game with the choosing rule "prior" (specifying the strategy to play in case of tie) for which the SPTD for even occurrences of the states is met, is a periodic game. This is applicable for the $M G^{\text {prior }}$ in the region of validity of SPTD for even occurrences, and for the $F S M G^{\text {prior }}$ in general.

Let us first assume the following simplified scenario. If SPTD for even occurrences is met for a MG game, then consider the following scoring rule for rewarding strategies: if a strategy rightly predicts the minority side in an odd occurrence of a state $\mu$ then it is rewarded with one point, and if the strategy wrongly predicts the minority side in an even occurrence of a state $\mu$ then one point is removed from its score $[14,10]$. This rule allows us to make a remarkable analytic simplification of the game, since the number of virtual points accumulated for any strategy at any time step ranges from 0 to $\mathcal{H}$.

For an agent $J$ of the $F S M G^{\text {prior }}$ whose two strategies are $\left(e_{1}^{J}, e_{2}^{J}\right)$, let us denote the virtual points accumulated for her set of strategies by $\left(v_{1}^{J}, v_{2}^{J}\right)$. The choosing rule of the strategy to play can be formalized by a function $\mathcal{D}\left(v_{1}^{J}, v_{2}^{J}\right)$ that returns the best ranked strategy of both and, in the case of a tie, it returns her a priori favorite one, i.e., $e_{1}^{J}$. SPTD implies that the number of possible combinations of virtual points in a given step $k$ of the game for the set of strategies $\left(v_{1}^{J}, v_{2}^{J}\right)_{k}$ is finite (in fact, there are at most $(\mathcal{H}+1)^{2}$ combinations) for the agent $J$. In the following, we will denote $\sharp X$ as the size of the set $X$. In a realization of the game with an infinite number of steps, $\sharp \mathcal{S}_{\mathcal{E}}=\infty$, at least one state $\mu \in \mathcal{H}$ appears an infinite number of times in the succession of states $\mathcal{S}_{\mathcal{E}}$, because the number of states is finite. Due to the fact that there are both finite combinations of virtual points for the strategies per agent $J$ and finite number of agents $(\mathcal{N})$, a step $l$ exists $(k<l)$ in which the state $\mu_{l}$ coincides with $\mu_{k}$. In addition, for every player $J,\left(v_{1}^{J}, v_{2}^{J}\right)_{k}=\left(v_{1}^{J}, v_{2}^{J}\right)_{l}$ is verified. Given that in case of tie, the choosing rule is not random, then $\mathcal{D}\left(v_{1}^{J}, v_{2}^{J}\right)_{k}=\mathcal{D}\left(v_{1}^{J}, v_{2}^{J}\right)_{l}$ and all the 
agents use the same strategy for $l$ which they had used for $k$. That is why they choose the same side in both stepts $k$ and $l$, and as a consequence, the minority side results the same in both $k$ and $l$ steps. Therefore, given $\left(v_{1}^{J}, v_{2}^{J}\right)_{k}=\left(v_{1}^{J}, v_{2}^{J}\right)_{l}$, the next step yields $\left(v_{1}^{J}, v_{2}^{J}\right)_{k+1}=\left(v_{1}^{J}, v_{2}^{J}\right)_{l+1}$. On the other hand, applying the same argument, from $\left(v_{1}^{J}, v_{2}^{J}\right)_{k+1}=\left(v_{1}^{J}, v_{2}^{J}\right)_{l+1}$ one gets $\left(v_{1}^{J}, v_{2}^{J}\right)_{k+2}=\left(v_{1}^{J}, v_{2}^{J}\right)_{l+2}$, and so on. Since the minority game with the prior choosing rule to the strategy to play (both $F S M G^{\text {prior }}$ and $M G^{\text {prior }}$ ) is deterministic, the game is periodic.

Note that the previous argument only relies on the facts that virtual points of strategies range between 0 and $\mathcal{H}$ (taking into account the SPTD) and that the decision function $\mathcal{D}$ is not random in case of tie (taking into account the choosing rule of $M G^{\text {prior }}$ ). Moreover, if the period length is $P$, then it is easy to see that the agents voting a given side in step $l+P$ are exactly the same agents than in step $l$. We call this fact strong periodicity, which obviously implies the periodicity of the minority side, an the periodiciy of the sequence of $N_{1}$. These results are consistent with the evidence observed in simulation of the $M G^{\text {prior }}$ game showed in previous section.

\section{General properties met by periodic SPTD sequences}

A directed graph $G=(V, E)$ is strongly connected if for each pair of vertices $v, u \in V, u \neq v$, there exists a directed path from $v$ to $u$ and a directed path from $u$ to $v$. Let us consider the operator $T: \mathcal{S}_{\mathcal{H}} \times\{0,1\} \rightarrow \mathcal{S}_{\mathcal{H}}$ that, given the current state $\mu=a_{1} a_{2} \ldots a_{m}$ and the new minority side $b$, the resulting state is defined by $T(\mu, b)=a_{2} a_{3} \ldots a_{m} b$. In other words, $T$ gives the state which follows a particular state $\mu$ (i.e., the successor of the state $\mu$ ) when the new minority side is $b$. The $M G$ graph for the $m$ parameter is the graph $G=(V, E)$ where $V=\mathcal{S}_{\mathcal{H}}$ (the set of states) and $E=\{(\mu, T(\mu, i)): \mu \in V, i=0,1\}$. This graph corresponds to the De Bruijn graph of order $m$, usual to analyse $\mathrm{MG}$ outcomes $[7,11]$. Note that every vertex of $G$ has indegree and outdegree equal to 2 , i.e., each state admits exactly two successors and exactly two predecessors) in $G$. We now state the following theorems about the behavior of periodic games which meet SPTD for even occurrences of the states using the $M G$ graph.

(1) The MG graph is strongly connected. 

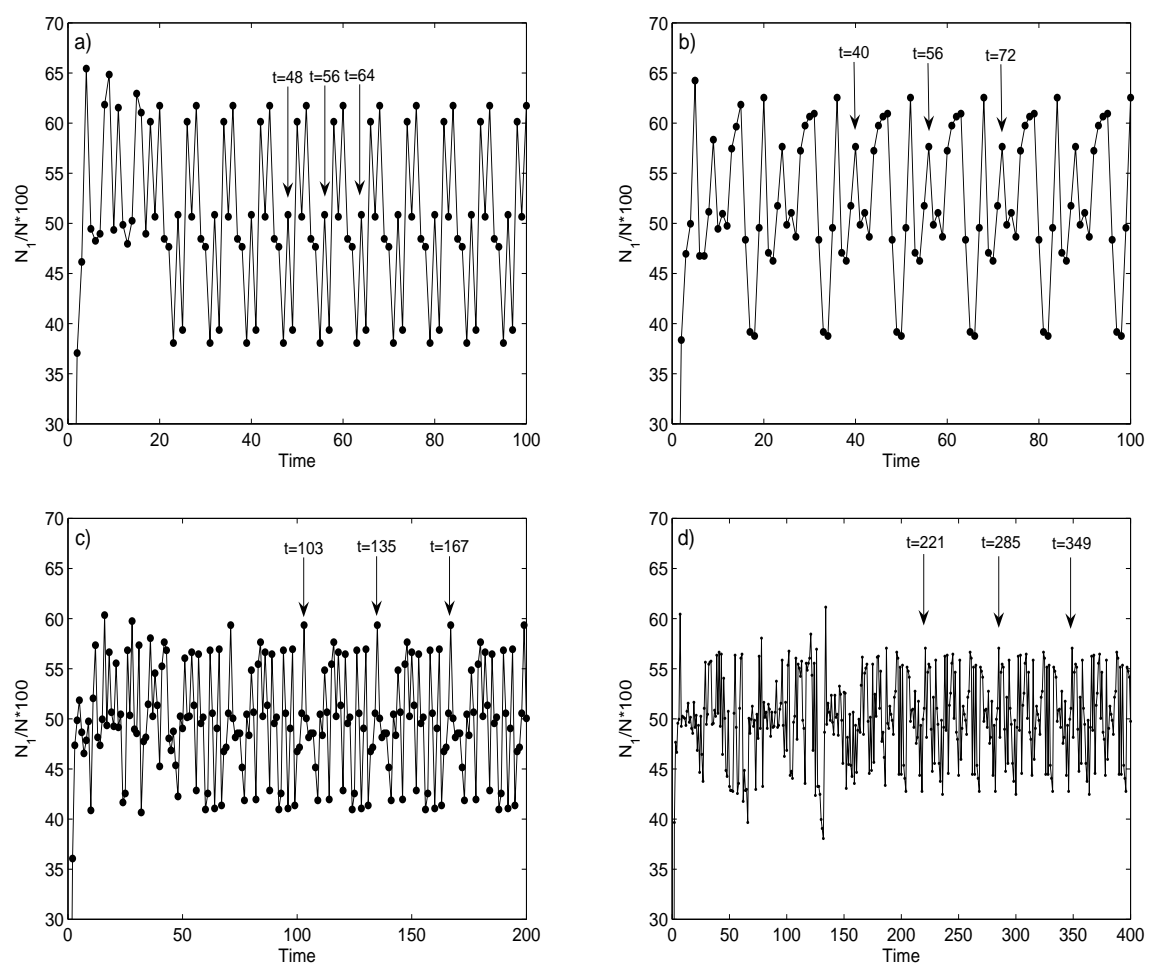

Figure 5: Periodicity of the sequence of the values of $N_{1}$ (equivalently, numbers of agents choosing side 0) for the $M G^{\text {prior }}$. The figures show periods of length a) 8, b) 16 , c) 32 , and d) 64 in games with $N=1001$ agents for values of $m=2,3,4$ and 5 , respectively. 


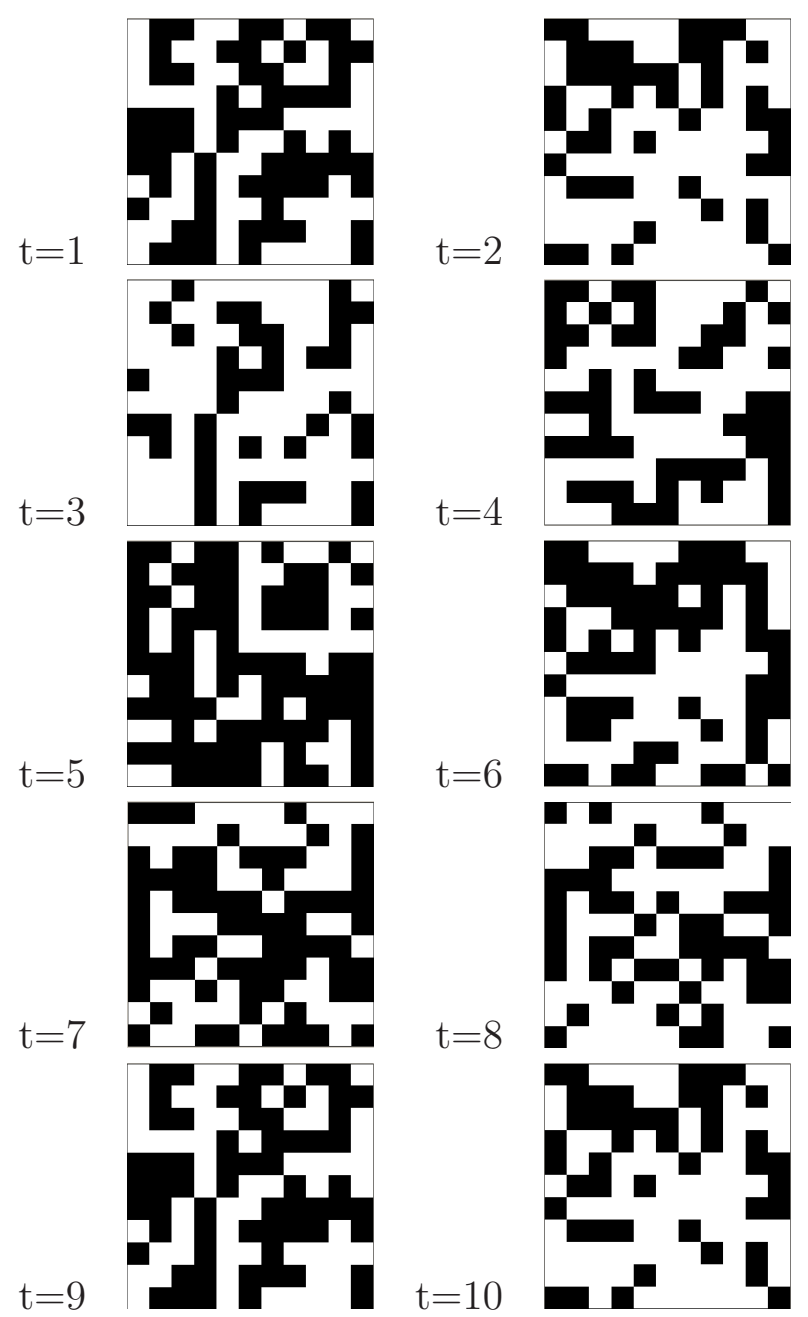

Figure 6: Strong periodicity: each pixel corresponds to the side chosen by an agent, black for side 1 and white for side 0 . The simulation corresponds to $m=2$ and $N=121$ agents, and the images correspond to 10 consecutive steps of the game. In this case, the game is periodic with period $P=8$, so the image for $t=1$ coincides with the image for $t=9$, and the image for $t=2$ coincides with the image for $t=10$. 
Since any sequence of $m$ bits can be obtained from any other after a finite number of iterations, the $M G$ graph is strongly connected.

(2) In the sequence of minority sides from the $M G$ of infinite size which meet SPTD in even occurrences of the states, all the states appear an infinite number of times.

We define the set $\mathcal{S}_{\mathcal{E}}$ to be the sequence of states appearing in a given realization $\mathcal{E}$ of the $M G$. Since $\mathcal{S}_{\mathcal{E}}$ is infinite and the $M G$ graph is strongly connected, then at least one state, say $\mu$, appears an infinite number of times. In particular, from the SPTD in even occurrences of the states, the successors of the state $\mu$, namely $T(\mu, 0)$ and $T(\mu, 1)$, also appear an infinite number of times. By applying the same argument to the successors, we conclude that in the sequence of states $\mathcal{S}_{\mathcal{E}}$, all states from $\mathcal{S}_{\mathcal{H}}$ appear an infinite number of times.

Theorems (3), (4), and (5) assume that the sequence is both periodic and that the SPTD is met for even occurrences of the states.

(3) If the $M G$ is periodic and meets the SPTD for the even occurrences of the states, then every state appears an even number of times in the period.

Let $\mu$ be a state and let $t$ be the number of times that $\mu$ appears in the period. If $t=1$ then $\mu$ has different occurrence parity in each two consecutive periods and the minority side in each period should swap (because of the SPTD), a fact which contradicts the periodicity of the $M G$. Suppose now that $t$ is odd and $t \geq 3$, and consider two consecutive periods. The even occurrences of $\mu$ in the first period will become odd occurrences in the second one and viceversa, which implies that there will be at least one alternance of minority sides between periods. However, if $\mu^{k}$ denotes the state in step $k$ and $P$ is the period length, then $\mu^{k}$ and $\mu^{k+P}$ must have the same minority result (by the periodicity), which shows that $t$ cannot be odd, a contradiction.

(4) If the $M G$ is periodic and meets the SPTD for the even occurrences of the states, then all the states appear in the period the same even number of times, which implies the ergodicity of the game.

In fact, let $\mu$ be the most frequently appearing state per period and let $c$ be the number of times it appears. As $c$ is even, we can write 
$c=2 k$, with $k \in \mathbb{N}$. Let $\mu_{0}$ and $\mu_{1}$ be the two preceding states of $\mu$, so that $T\left(\mu_{0}, 0\right)=T\left(\mu_{1}, 1\right)=\mu$. On the one hand, if a state $\tilde{\mu}$ appears in a period $\tilde{c}$ times preceding a minority side $\tilde{o}$ then it appears another $\tilde{c}$ times preceding the opposite minority side $\sim \tilde{o}$. This is explained because, without lost of generality, taking the period as starting from an odd occurrence of $\tilde{\mu}$, the SPTD implies a minority side $\sim \tilde{o}$ per any side $\tilde{o}$. On the other hand, $\mu$ appears $2 k$ times and is preceded only by $\mu_{0}$ and $\mu_{1}$. Let us call $k_{0}$ (resp. $k_{1}$ ) the number of times the state $\mu_{0}$ (resp. $\mu_{1}$ ) appears preceding $\mu$. Then $k_{0}+k_{1}=c$ (the argument includes the case in which $\mu_{0}=\mu$ or $\mu_{1}=\mu$ ) and we claim that $k_{0}=k_{1}=c / 2=k$. Otherwise, we could suppose that $k_{0}>c / 2$, but if $\mu_{0}$ appears $k_{0}$ times preceding $\mu$ (hence a given minority side $\tilde{o}$ ), and then another $k_{0}$ times preceding $\sim \tilde{o}$, we get that $\mu_{0}$ appears at least $2 k_{0}>2 k=c$ times, a contradiction since $c$ is the highest number of times that a given state appears in a period. Therefore, $k_{0}=k_{1}=c / 2$ and both predecessors of $\mu$ appear $c$ times per period. Taking into account that the graph is strongly connected, the same argument can be applied to each state of the period.

From the previous results we obtain that if we have a periodic game which meets the SPTD for even occurrence of states, then all the states of the set $\mathcal{S}_{\mathcal{H}}$ appear the same even number of times. Thus the period $P$ can be written as $P=2 k \mathcal{H}=k 2^{m+1}$.

(5) If the $M G$ is periodic and meets the SPTD for the even occurrences of the states, then it also meets the SPTD for the odd occurrences if and only if the period is $P=2 \mathcal{H}=2^{m+1}$.

Let us first prove the converse implication. In fact, if $P=2 \mathcal{H}=2^{m+1}$ and knowing that the number of states is $\mathcal{H}=2^{m}$ and all of them appear the same number of times, we conclude that such a number of times equals two. Let us build the period in such a way that a given state $\mu$ appears first in an odd occurrence with resulting minority side $\tilde{o}$. Then, the SPTD for even occurrences implies that the next occurrence (which is even and lies within the same period) corresponds to the minority side $\sim \tilde{o}$. The next occurrence of $\mu$ is again an odd occurrence (there are two states per period) and the periodicity implies that the resulting minority side will be $\tilde{o}$. Hence, minority sides alternate in each 
occurrence of $\mu$, which implies the SPTD is met for odd occurrences.

For the forward implication, suppose that SPTD is valid for both even and odd occurrences of the states. The SPTD in odd and even occurrences implies alternances in the minority sides for any given state.

Let us select a state $\mu$ with the minimum number of steps between three consecutive occurrences within a period. Let $\tilde{\mu}^{k}=\tilde{\mu}^{l}=\tilde{\mu}^{s}=\mu$ be such consecutive occurrences of $\mu$, with $k<l<s$. In this setting, $s-k$ is the smallest possible separation between three repetitions of a state.

Note that the preceding states of $\mu^{l}$ and $\mu^{s}$ (i.e., $\tilde{\mu}^{l-1}$ and $\tilde{\mu}^{s-1}$ ) must be different from each other. Otherwise, if $\tilde{\mu}^{l-1}=\tilde{\mu}^{s-1}$, there must be another occurrence of the state $\tilde{\mu}^{l-1}$ between the former ones due to the fact that no state can be followed by the same minority side in two consecutive occurrences. But this is impossible because $s-k$ is the smallest separation between three consecutive repetitions of any state. We conclude that $\tilde{\mu}^{l-1} \neq \tilde{\mu}^{s-1}$. As a consequence, the preceding state of $\tilde{\mu}^{s}$ is the same preceding $\tilde{\mu}^{k}$, taking into account that SPTD is valid in both even and odd occurences, and that the only appearances of the state $\mu$ between the steps $k$ and $s$ are in the steps $k, l$ and $s$.

Now, as $\tilde{\mu}^{k-1}=\tilde{\mu}^{s-1}$, then $\tilde{\mu}^{k-1}$ must necessarily appear exactly once between the steps $k-1$ and $s-1$, in order not to be followed by the same minority side twice in a row.

By repeating this argument with the preceding states of $\tilde{\mu}^{k-1}$ and $\tilde{\mu}^{s-1}$, we can see that in a finite number of steps we will attain the state $\tilde{\mu}^{k}$. Also, we obtain that the sequence between $k$ and $s-1$ is previously repeated in exactly the same way. Formally, if $s-k=L$, then $\mu^{t}=\mu^{t-L}$ for $t=k, \ldots, s-1$. As this sequence of size $L$ is repeated infinitely, then this sequence corresponds to some multiple of the period of minority sides, which we know to be of size $P=2 k \mathcal{H}$. Then, $L=2 j \mathcal{H}$, with $j / k \in \mathbb{N}$. On the other hand, in this sequence of size $L$, each state appears exactly twice (i.e., those states which do appear, though we do not know yet if all the states of the game are present in the sequence). Therefore, $L$ must have size at most $2 \mathcal{H}$. As a consequence, since $L \leq 2 \mathcal{H}$ and $L=2 j \mathcal{H}$, then $j=1$ and $L=2 \mathcal{H}$.

Thus, a periodic sequence of a periodic MG which meets SPTD for even occurences results in an eulerian path in the De Bruijn graph of order $m$, and 
in a hamiltonian path in the De Bruijn graph or order $m+1$. The number of different eulerian paths in a De Bruijn graph of order $m$ (established in [11]) can be obtained using the BEST Theorem [17]:

$$
\frac{2^{2^{m}}}{2^{(m+1)}}=\frac{\mathcal{L}}{2 \mathcal{H}}
$$

It is remarkable that the amount of eulerian paths (hence the amount of different periodic sequences) can be written as a function of the size of the set of strategies $\mathcal{L}$ and the size of the set of states $\mathcal{H}$. This fact poses the question whether there exists any deeper relation between the number of eulerian paths in the De Bruijn graph and the relevant parameters of the MG.

Previous results are mostly abstract in the sense that they extract conclusions from the SPTD and the periodicity of minority sides only. This means that any infinite chain of minority sides generated by any method (not necessarily the $M G$ ) must verify these facts. In particular, we have already observed by numerical simulations that the $M G^{\text {prior }}$ is periodic along the first part of the symmetric phase. This fact has been shown in Section 2, where we present the outcomes of minority sides for the $M G^{\text {prior }}$ model for $m=2$. These results are in accordance with the theorems stated in the present section. In Section II we have shown two possible periodic sequences of length $L=8=2 \mathcal{H}$ which occur in most of the realizations of the $M G^{\text {prior }}$ in which the SPTD is met for even and odd occurrences of the states, in accordance with Theorem (5). Furthermore, the fact that there are two of these sequences for $m=2$ agrees with Eq. (1). The other periodic sequences appearing in the realizations consigned in Table 2 (i.e., realizations with period length $L>2 \mathcal{H}$ ) do not meet the SPTD for odd occurrences while they do meet SPTD for even occurrences of the states. Note that the length of these periodic sequences always has the form $L=2 k \mathcal{H}$, with $k$ an integer value greater than 1 (hence $L=16$ and $L=24$ for these cases). These results are in accordance with Theorems (4) and (5).

Similar arguments can be applied to different deterministic versions of the $M G$. We briefly explore the cases emerging from the following choosing rules in case of tied strategies:

- Rule 1: the agent uses the same strategy that she has used in the previous step of the game. 
- Rule 2: the agent chooses the opposite side that she has chosen the previous time that she was undecided.

- Rule 3: the agent plays the opposite strategy that she has played the previous time that she was undecided.

In all these cases, the outcome obtained in simulations turns out to be periodic for small values of $\alpha$ yielding periods of the size predicted by the theory. SPTD is met for even occurrences in all the cases, as Figure 7 shows. This fact and the deterministic rule in case of ties ensure that the outcome of the game is periodic, as we shown in Section 3. Indeed, for $m=2$, sequences of period $L=16$ are obtained for rule 1 and $L=8,16$ for both rules 2 and 3. For $m=2$, SPTD for odd occurrences of the states is met the majority of the runs performed for the switching rules 2 and 3 (whose outcome sequence results in the known 00011101 and 11100010), while SPTD for odd occurrences of the states is never met in runs of repeating rule 1 , whose outcomes have period length $L=16$. Figure 8 compares the values of $\sigma^{2} / N$ for the $M G$ and for the new rules:

- In panel a) we show the $M G$ and the $M G^{\text {prior }}$ case, which shows all values of $\alpha$ as we has just discussed.

- In panel b) we show results for rule 1, which are greater than those of the $M G$ case for values of $\alpha \rightarrow 0$.

- In panels c) and d) we show results for rule 2 and 3 respectively, which concur with the $M G$ case too.

This last fact and the similarity in the PTD for odd occurrences of the states show that the $M G^{\text {prior }}$ and the $M G$ with rules 2 or 3 are more adecuate to shed light about the $M G$.

As another application, we briefly analize the case of the $M G^{\text {prior }}$ in which an initial bias is introduced to the strategies scores. As made in [10] with the $M G$ case, the bias is introduced at the agents level, i.e., each agent randomly chooses - with a bias probability $p_{b}$ - to assign "a priori" $u_{o}$ virtual points to any of her strategies. For the sake of simplicity, we fix the bias probability to be $p_{b}=1 / 2$. In [10] analytic results for the $F S M G$ with biased scores are presented, in particular showing that SPTD is met for the FSMG model for all values of $u_{o}$. In Figure 9 simulations for the $M G^{\text {prior }}$ with biased scores are shown, reflecting values of $\sigma^{2} / N$ smaller than in the 

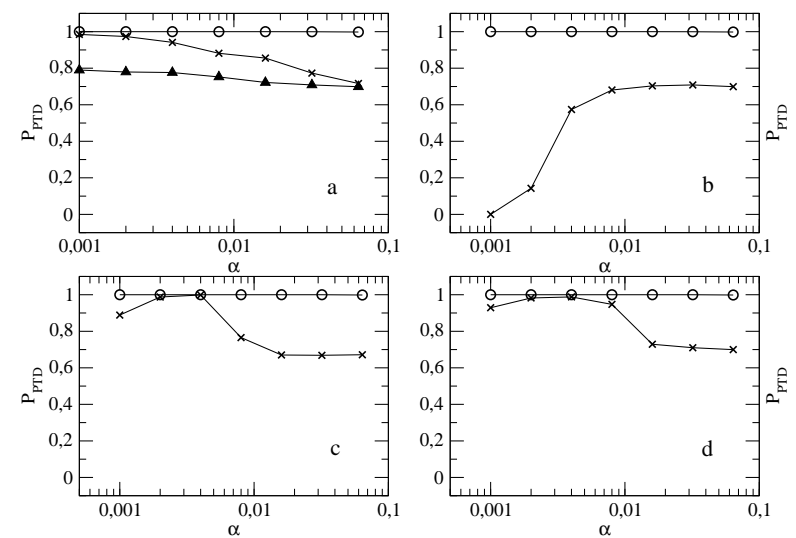

Figure 7: Probability $P_{P T D}$ for PTD to take place in even (circles) and odd (X) occurrences of the states as a function of $\alpha$, calculated as 1 minus the probability of breaking the PTD for the first time in a given simulation of the game for different values of $m$ (from 2 to 7 ) and $N=4001$. Panel a) contains the $M G^{\text {prior }}$ and $M G$ case (up triangle for odd occurrences of $M G$ ); panel b) shows the $M G$ with rule 1 (undecided agents use the same strategy as the previous step); panel c) contains the $M G$ with rule 2 (undecided agents systematically switch the minority side in case of tie); and panel d) shows results for the $M G$ with rule 3 (undecided agents systematically switch the strategy to play in case of tie). For each value of $m, 100$ runs have been performed, each one of $T=100000$ time steps discarding the first 50000 steps.

biased $M G^{\text {prior }}$ depending of the biased value $u_{o}$, while the SPTD is broken in even occurrences of the states. Indeed, for $m=2$ we observed that in simulations for different values of $u_{o}$ from 2 to $20, S P T D$ is valid for even and odd occurrences of the states and the periodic sequences obtained are the two known sequences associated with the eulerian cycles of the De Bruijn graph of order 2, namely 00011101 and 11100010.

The reason for the periodicity of $M G^{\text {prior }}$ is its deterministic choosing rule of the strategies $\mathcal{D}$, in addition to the validity of the SPTD for even occurrences of the states along the first part of the symmetric phase. Related to these observations, in Appendix A we prove that the $F S M G^{\text {prior }}$ necessarily meets the SPTD for even occurrences of the states. 

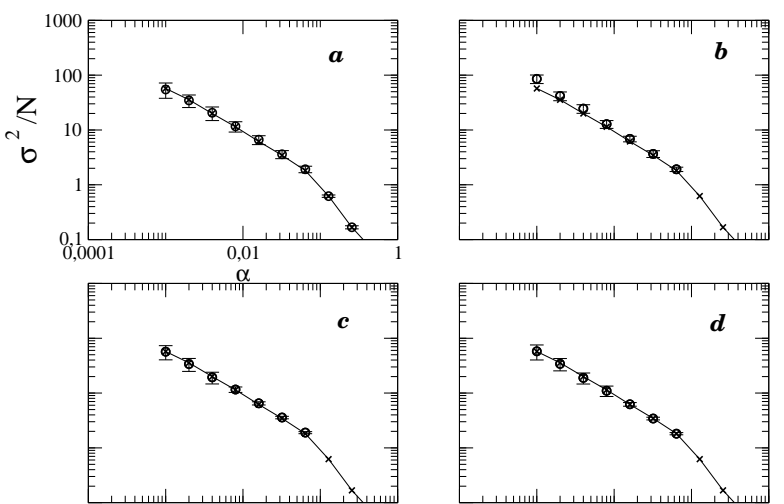

Figure 8: The $\mathrm{X}$ symbols show $\sigma^{2} / N$ as a function of $\alpha$ for the $M G$ for different values of $m$ (from 2 to 7 ) and $N=4001$, and the circular symbols contain the same measurements for the $M G$ with alternative choosing rules in case of tie. Panel a) shows the results for the $M G^{\text {prior }}$; panel b) shows results for the $M G$ with rule 1 (undecided agents use the same strategy as the previous step); panel c) contains results for the $M G$ with rule 2 (undecided agents systematically switch the minority side in case of tie); and panel d) reports the results for the $M G$ with rule 3 (undecided agents systematically switch the strategy to play in case of tie). For each value of $m, 100$ runs have been performed, each one of $T=100000$ time steps discarding the first 50000 steps. Online error bars of circle symbols (deterministic models) are shown. Scales and labels are the same in the four panels. 


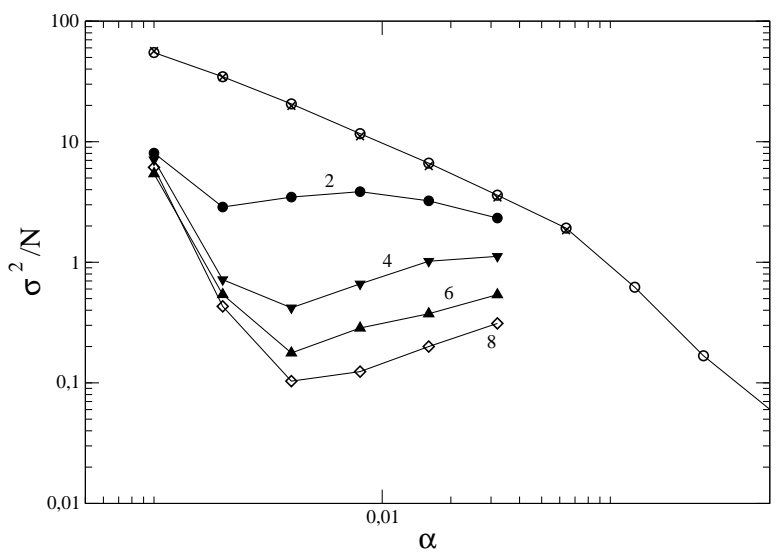

Figure 9: The $\mathrm{X}$ symbols show $\sigma^{2} / N$ as a function of $\alpha$ for the $M G$ for different values of $m$ and $N=4001$, and the other symbols contain the same measurements for the $M G^{\text {prior }}$. White circles correspond to the $M G^{\text {prior }}$; filled circles correspond to the biased case with bias $u_{o}=2$; up triangles correspond to $u_{o}=4$; down triangles correspond to $u_{o}=6$; and diamonds correspond to $u_{o}=8$. For each value of $m, 100$ runs have been performed, each one of $T=100000$ time steps discarding the first 50000 steps. 


\section{Description of the quasi-periodicity of the MG for $m=2$}

It is known that the MG is not deterministic as the $M G^{\text {prior }}$ model. The periods appearing in the $M G^{\text {prior }}$ turn out to be quasi-periods in the $\mathrm{MG}$ realizations, as can be seen from the wide variety of graphs generated in this case. For $m=2$, the two periodic sequences of length $L=2 \mathcal{H}=8$ from the $M G^{\text {prior }}$ are present in the graphs associated with the $\mathrm{MG}$, although additional nodes are present too, because the outcomes of the MG partially travel these periodic sequences but also take other paths before returning to one of the two periodic sequences. In this section we provide an empirical description of these quasi-periods for $m=2$, based on a set of 100 realizations of the MG for $N=1001$ agents.

Consider the two eulerian cycles associated with the sequences 00011101 and 11100010 and the eight nodes of each one. When we observe the outcomes of the MG, for each node of these eulerian cycles there are two possibilities, according to the minority side of the outcome after the sequence of size $\mathcal{H}$ : either to continue to the next node within the same eulerian cycle or to frustrate the cycle towards an out-of-cycle node. The latter admits two further possibilities: either frustration breaks towards an inner path, which eventually returns to the same cycle, or frustration takes an outer path, which ends up in the other eulerian cycle. In realizations when outer paths are present, the two eulerian cycles are shown. We call $E 1$ the cycle in Figure 4.a (i.e., corresponding to the sequence 00011101), and we call E2 the cycle in Figure 4.b (i.e., corresponding to the sequence 11100010). Note that each node of a cycle has a symmetric opposite in the other cycle, which is obtained by swapping sides 0 and 1 (for example, the symmetric opposite of the node 01110100 is the node 10001011). Moreover, each node has an inverse node in the other cycle, which is obtained by reading the 8 bits in reverse, from back to front (thus, the inverse of the node 01110100 is the node 00101110). From numerical simulations we found that both inner and outer paths are fixed, that is, for each eulerian cycle there are 4 inner paths and 4 outer paths beginning in nodes from the cycle and ending in nodes of the other cycle. We have empirically observed that inner paths show the following features:

- The four inner paths of each eulerian cycle are grouped in two pairs. In fact, they appear in pairs in MG simulations, each member of a pair having a similar frequency of occurrence. In $E 1$, the first pair is composed by the two inner paths beginning in the nodes ending in 
11, namely $101000 \underline{11}$ and $010001 \underline{11}$ (e.g., in Figure 4.c only these two inner paths appear). The second pair is given by the two inner paths beginning in the nodes ending in 00, namely $011101 \underline{00}$ and $111010 \underline{00}$. For example, in Figure 7.a the four inner paths of $E 1$ are present, although the second pair is less frequent than the first one. By the symmetry between the two eulerian cycles, the same occurs for $E 2$ : the first pair is given by the inner paths beginning in the nodes ending in 11, namely $100010 \underline{11}$ and $000101 \underline{11}$ (as Figure 7.b shows), and the second pair is given by the inner paths beginning in the nodes ending in 00, namely 010111미 and $101110 \underline{00}$ (as Figure 7.c shows). The graph in Figure 7.d contains all four inner paths of $E 2$.

- All the inner paths have length 6 , so they traverse through 5 intermediate nodes.

In turn, outer paths connecting $E 1$ and $E 2$ show the following features:

- The frustrating node of a cycle is the inverse (reading backwards) of the arriving node in the other eulerian cycle, and the path has the minimum number of steps needed to go from the 8-bit starting node to its inverse. For example, in Figure 8.a there are two outer paths of length $L=5$, the first one beginning in node 00111010 from $E 1$ and ending in node 01011100 from $E 2$, and the second one beginning in node 11100010 from $E 2$ and ending in node 01000111 from $E 1$. In Figure 4.d there are two outer paths of length $L=3$, the first one beginning in node 11010001 from $E 1$ and ending in node 10001011 from $E 2$, and the second one beginning in node 01110001 from $E 2$ and ending in node 10001110 from E1. In Figure 8.b there are two outer paths of length $L=3$. Note that the path beginning in node 00101110 from $E 2$ and ending in node 01110100 from $E 1$ is the same path appearing in Figure 4.c but traversed in the opposite direction.

- Two outer paths of length 5 leave each eulerian cycle, and two outer paths of the same length arrive the cycle. Two further outer paths of length 3 leave the cycle, and two outer paths of the same length arrive the cycle.

- The outer paths frustrate the cycles in nodes ending in 01 and 10. 

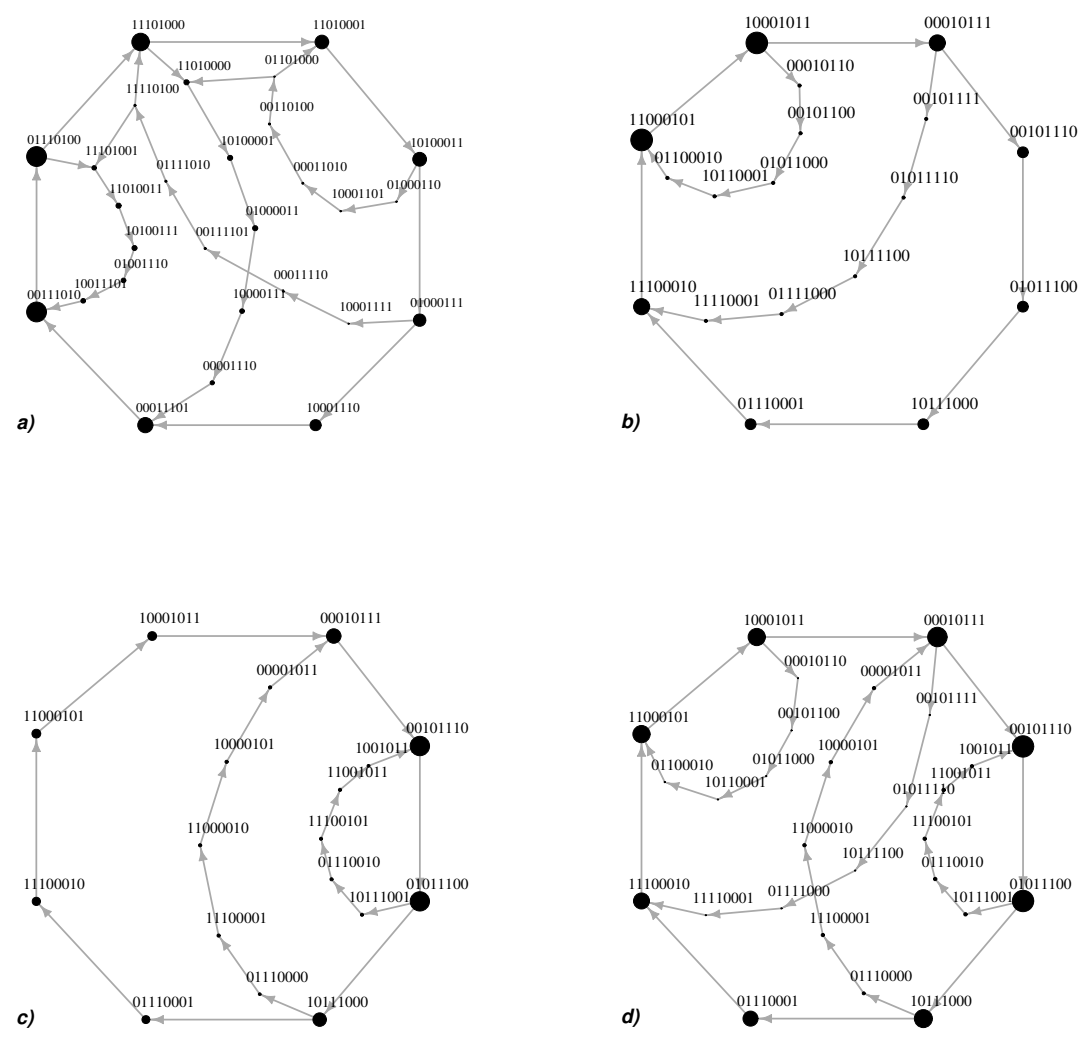

Figure 10: Eulerian paths and different inner paths in four $M G$ realizations. In all cases there are $N=1001$ agents, we set $m=2$, and we perform 2000 steps of the game in the $M G$ case. 

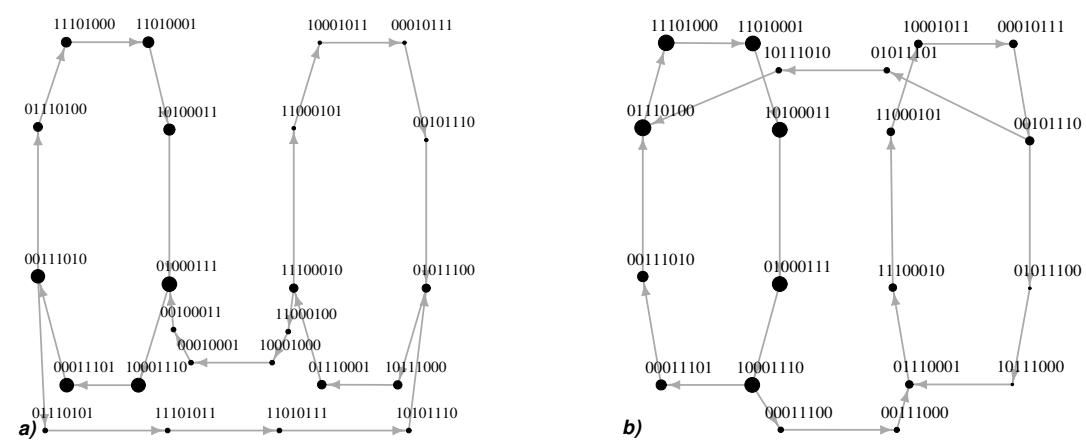

Figure 11: Eulerian paths and different outer paths in two $M G$ realizations. In all cases there are $N=1001$ agents, we set $m=2$, and we perform 2000 steps of the game in the $M G$ case.

\section{Conclusions}

In this work we proposed the $M G^{\text {prior }}$, which clarifies the quasi-periods observed in the sequence of minority sides of the $M G$. In this new model, agents use their favourite strategy in case of tie, thus generating a deterministic execution. We have shown that in the SPTD regime for even occurrences of the states, the outcomes of the $M G^{\text {prior }}$ are a periodic sequence and, moreover, the decisions of the agents are also periodic (strong periodicity). Furthermore, we have proposed the $F S M G^{\text {prior }}$, a maximal instance of the $M G^{\text {prior }}$ in which all the potential agents are present (in the same way that the FSMG was defined in [10]). By exploiting the symmetry of the $F S M G^{\text {prior }}$, we showed that the $F S M G^{\text {prior }}$ necessarily verifies the SPTD. In Appendices $\mathrm{A}$ and $\mathrm{B}$ we prove the equivalence between the $F S M G_{\text {rand }}^{\text {prior }}$ and the $M G_{\text {rand }}^{\text {prior }}$ in terms of $\sigma^{2} / N$.

We have proved some general theorems applicable for sequences which meet periodicity and SPTD for even and/or odd occurrences of the states (i.e., not necessarily coming from a minority game). These theorems imply that in the regime in which SPTD is met for even occurences of the states, the sequence of minority sides of the $M G^{\text {prior }}$ results periodic with length $L=$ $2 k \mathcal{H}$, and $k=1$ when the SPTD is met for both even and odd occurrences. 
In these cases, we showed that the periodic sequences for the $M G^{\text {prior }}$ with parameter $m$ are obtained as the eulerian cycles in the De Bruijn graph of order $m$. For example, when $m=2$ there are two eulerian cycles associated with the periodic sequences 11100010 and 00011101. We have characterized the quasi-periods of the MG for $m=2$ as deviations from these eulerian cycles. These deviations sometimes generate inner paths which end in the same eulerian cycle, and sometimes generate outer paths which end in the other eulerian cycle.

It is known that $P_{P T D} \sim 1$ for even occurrences of the states of the $M G$ in the symmetric phase. However, we showed that $P_{P T D}$ is much greater than 0.5 (approx. 0.8) for odd occurrences of the states in the same phase for the $M G$. Remarkably in both cases (i.e., the periods in the $M G^{\text {prior }}$ and the quasi-periods in the $M G$ ) the PTD of odd occurrences of a states is not accompanied by crowd effects.

Finally, we conclude that the fact that the sequence of outcomes is not periodic in the MG is generated by the random breaking of tied strategies, following the original choosing rule of strategies of the $M G$.

\section{Appendix A. SPTD and FSMG}

As defined in the main text and according to the notation in [10], $\mathcal{S}_{\mathcal{H}}$ and $\mathcal{S}_{\mathcal{L}}$ denote the set of states and strategies respectively, which coincide with the Full Strategy Space. Symbol $\sharp$ stands for the cardinality of a set, hence $\sharp \mathcal{S}_{\mathcal{H}}=\mathcal{H}$, and $\sharp \mathcal{S}_{\mathcal{L}}=\mathcal{L}$.

For an arbitrary outcome $\tilde{o} \in\{0,1\}$, we will denote the opposite side by $\sim \tilde{o}$. For a given state $\mu \in \mathcal{S}_{\mathcal{H}}$, the subset of strategies in $\mathcal{S}_{\mathcal{L}}$ which predict a certain outcome $\tilde{o}$ for the state $\mu$ is denoted by $\mathcal{S}_{\mathcal{L}, \mu \rightarrow \tilde{o}}$. It is clear that $\mathcal{S}_{\mathcal{L}, \mu \rightarrow \tilde{o}} \cup \mathcal{S}_{\mathcal{L}, \mu \rightarrow \sim \tilde{o}}=\mathcal{S}_{\mathcal{L}}$, and that $\sharp \mathcal{S}_{\mathcal{L}, \mu \rightarrow \tilde{o}}=\sharp \mathcal{S}_{\mathcal{L}, \mu \rightarrow \sim \tilde{o}}=\mathcal{L} / 2$.

The main idea in [10] consists in defining an ensemble of states of the $F S M G$ for which it is possible to analytically compute the distribution of virtual points accumulated for all the strategies of $\mathcal{S}_{\mathcal{L}}$. In particular, $\mathcal{S}_{\mathcal{L}, l}$ denotes the set of strategies with $l$ virtual points, and $\mathcal{S}_{\mathcal{L}, \mu \rightarrow \tilde{o}, l}$ (resp. $\mathcal{S}_{\mathcal{L}, \mu \rightarrow \sim \tilde{o}, l}$ ) denotes the set of strategies with $l$ virtual points that predict $\tilde{o}($ resp. $\sim \tilde{o}$ ) for $\mu$. For each step $t$, the parity array $\mathcal{P}_{\mathcal{E}}^{t}$ is an array of elements from a categorical variable which take two possible values: $O$ and $E$ (odd or even), recording the parity of the number of appearences of each state in the first $t-1$ steps. More precisely, $\mathcal{P}_{\mathcal{E}}^{t} \in\{O, E\}^{\mathcal{H}}$. When we identify any state $\mu$ with the integer number given by the binary expansion of $\mu$ plus 1 (so that $\mu$ 
can be thought of as an integer ranging from 1 to $\mathcal{H}), \mathcal{P}_{\mathcal{E}}^{t}(\mu)=O$ (resp. $E$ ) if $\mu$ has appeared an odd (resp. even) number of times in the first $t-1$ steps of the game (see [10] for details). In [10] we had shown that the state is characterized by $\mathcal{P}\left(\mu_{p}\right)$ (the parity of the present state) and $n_{0}$ (the number of states for which $\mathcal{P}(\mu)=O)$.

In $[10]$ it is shown that

$$
\sharp \mathcal{S}_{\mathcal{L}, l}=\left(\begin{array}{c}
n_{o} \\
l
\end{array}\right) \mathcal{L} / 2^{n_{o}} .
$$

and, if $\mu$ is in an even state (i.e., $\mathcal{P}(\mu)=E$ ), then

$$
\sharp S_{\mathcal{L}, \mu \rightarrow \tilde{o}, l}=\sharp S_{\mathcal{L}, \mu \rightarrow \sim \tilde{o}, l}=\sharp S_{\mathcal{L}, l} / 2=\left(\begin{array}{c}
n_{o} \\
l
\end{array}\right) \mathcal{L} / 2^{n_{o}+1} .
$$

If state $\mu$ is in an odd state $(\mathcal{P}(\mu)=O)$, then

$$
\begin{aligned}
& \sharp S_{\mathcal{L}, \mu \rightarrow \sim \tilde{o}, l}=\left(\begin{array}{c}
n_{o}-1 \\
l
\end{array}\right) \mathcal{L} / 2^{n_{o}}, \\
& \sharp S_{\mathcal{L}, \mu \rightarrow \tilde{o}, l}=\left(\begin{array}{c}
n_{o}-1 \\
l-1
\end{array}\right) \mathcal{L} / 2^{n_{o}} .
\end{aligned}
$$

All the calculations in [10] with respect to the set of strategies are applicable here, but not the calculations involving the set of agents, which must be recalculated (for example, according to the definition of the FSMG ${ }^{\text {prior }}$, $\mathcal{N}_{u}=0$, because there are no undecided agents in the $\left.M G^{\text {prior }}\right) . \mathcal{N}_{\tilde{o}}$ and $\mathcal{N}_{\sim \tilde{o}}$ represent the number of agents choosing the option $\tilde{o}$ and the opposite option $\sim \tilde{o}$, so that $\mathcal{N}_{\tilde{o}}+\mathcal{N}_{\sim \tilde{o}}=\mathcal{N}$. For a particular state of the ensemble, for which the present system state is $\mu_{p}, \mathcal{N}_{\tilde{o}}$ can be written as

$$
\begin{gathered}
\mathcal{N}_{\tilde{o}}=2 \sum_{l=1}^{n_{o}} \sharp \mathcal{S}_{\mathcal{L}, \mu_{p} \rightarrow \tilde{o}, l}\left(\sum_{j=0}^{l-1} \sharp \mathcal{S}_{\mathcal{L}, \mu_{p} \rightarrow \sim \tilde{o}, j}\right)+ \\
\sum_{l=0}^{n_{o}} \sharp \mathcal{S}_{\mathcal{L}, \mu_{p} \rightarrow \tilde{o}, l} \sharp \mathcal{S}_{\mathcal{L}, \mu_{p} \rightarrow \sim \tilde{o}, l}+\left(\sharp \mathcal{S}_{\mathcal{L}, \mu_{p} \rightarrow \tilde{o}}\right)^{2}
\end{gathered}
$$

The first term in the previous expression computes all the pairs in which the most successful strategy predicts $\tilde{o}$ and the least successful predicts $\sim \tilde{o}$, 
regardeless of the order of the strategies in the ordered pair. The second term computes the pairs of strategies which, having accumulated equal numbers of points and predicting different sides, are ordered in such a way that the first (favorite) strategy of the pair is the one predicting $\tilde{o}$, and the second strategy is the one predicting $\sim \tilde{o}$. This means that it is the a priori choice which leads these agents to play side $\tilde{o}$. The last term computes all the pairs of strategies in which both strategies predict side $\tilde{o}$. By symmetry, there will be an equal term for the agents who will play side $\sim \tilde{o}$ :

$$
\begin{aligned}
& \mathcal{N}_{\sim \tilde{o}}=2 \sum_{l=1}^{n_{o}} \sharp \mathcal{S}_{\mathcal{L}, \mu_{p} \rightarrow \sim \tilde{o}, l}\left(\sum_{j=0}^{l-1} \sharp \mathcal{S}_{\mathcal{L}, \mu_{p} \rightarrow \tilde{o}, j}\right)+ \\
& \sum_{l=0}^{n_{o}} \sharp \mathcal{S}_{\mathcal{L}, \mu_{p} \rightarrow \tilde{o}, l} \sharp \mathcal{S}_{\mathcal{L}, \mu_{p} \rightarrow \sim \tilde{o}, l}+\left(\sharp \mathcal{S}_{\mathcal{L}, \mu_{p} \rightarrow \sim \tilde{o}}\right)^{2}
\end{aligned}
$$

It is easy to check, using Eq. (A.2), that $\mathcal{N}_{\tilde{o}}=\mathcal{N}_{\sim \tilde{o}}$ when the present state $\mu_{p}$ is such that $\mathcal{P}_{\mathcal{E}}^{t}\left(\mu_{p}\right)=E$. Thus, this new appearance of the state corresponds to an odd occurence of the state (i.e., up to the step $t-1$ it has appeared an even number of times).

Assume now that $\mathcal{P}_{\mathcal{E}}^{t}\left(\mu_{p}\right)=O$ and suppose that $\tilde{o}$ was the outcome of the game after the last (hence even) appearance of $\mu_{p}$. In this case, we use (A.4) and (A.3) to obtain $\mathcal{N}_{\tilde{o}}$ and $\mathcal{N}_{\sim \tilde{o}}$ :

$$
\begin{aligned}
& \mathcal{N}_{\tilde{o}}=\mathcal{L}^{2} / 2^{2 n_{o}-1}\left(\sum_{l=1}^{n_{o}}\left(\begin{array}{c}
n_{o}-1 \\
l-1
\end{array}\right)\left(\sum_{j=0}^{l-1}\left(\begin{array}{c}
n_{o}-1 \\
j
\end{array}\right)\right)\right)+ \\
& \mathcal{L}^{2} / 2^{2 n_{o}} \sum_{l=1}^{n_{o}-1}\left(\begin{array}{c}
n_{o}-1 \\
l-1
\end{array}\right)\left(\begin{array}{c}
n_{o}-1 \\
l
\end{array}\right)+\left(\begin{array}{c}
\mathcal{L} \\
2
\end{array}\right), \\
& \mathcal{N}_{\sim \tilde{o}}=\mathcal{L}^{2} / 2^{2 n_{o}-1}\left(\sum_{l=1}^{n_{o}-1}\left(\begin{array}{c}
n_{o}-1 \\
l
\end{array}\right)\left(\sum_{j=1}^{l-1}\left(\begin{array}{c}
n_{o}-1 \\
j-1
\end{array}\right)\right)\right)+ \\
& \mathcal{L}^{2} / 2^{2 n_{o}} \sum_{l=1}^{n_{o}-1}\left(\begin{array}{c}
n_{o}-1 \\
l-1
\end{array}\right)\left(\begin{array}{c}
n_{o}-1 \\
l
\end{array}\right)+\left(\begin{array}{c}
\mathcal{L} \\
2
\end{array}\right) .
\end{aligned}
$$


In short, if agents process a state in an even appearance, then

$$
\mathcal{N}_{\tilde{o}}-\mathcal{N}_{\sim \tilde{o}}=\mathcal{L}^{2} / 2^{2 n_{o}-1}\left(\begin{array}{c}
2 n_{o}-1 \\
n_{o}
\end{array}\right)>0
$$

which shows that FSMG ${ }^{\text {prior }}$ necessarily verifies the SPTD when the present state is in an even occurence (i.e., the parity function up to time step $t-1$ is $O$ and for this reason, the present state is occurring in an even appearance). Indeed, (A.9) has been obtained under the assumption that $\tilde{o}$ was the outcome of the game after the previous appearance of $\mu_{p}$, and shows that the minority side will be $\sim \tilde{o}$ after the agents have processed the new appearance of $\mu_{p}$. By symmetry, the same holds by changing $\tilde{o}$ by $\sim \tilde{o}$.

\section{Appendix B. $M G_{\text {rand }}$ and $M G_{\text {rand }}^{\text {prior }}$ : the same analytical results}

Once the FSMG $G^{\text {prior }}$ is solved, we can consider an instance $I$ of the $M G^{\text {prior }}$ as a random sample of size $N$ from the $\mathcal{N}$ agents of the FSMG ${ }^{\text {prior }}$. To this end and according to the ideas of [10], let us consider an experiment consisting in the random extraction of a sample size $N$ (with repetition) from a box containing $\mathcal{N}$ agents of two different types, namely $\mathcal{N}_{\tilde{o}}$ agents of type 1 and $\mathcal{N}_{\sim \tilde{o}}$ agents of type 2 . Suppose that after extracting the sample, we obtain $N_{\tilde{o}}$ agents of type 1 and $N_{\sim \tilde{o}}$ agents of type 2 , so that $N_{\tilde{o}}+N_{\sim \tilde{o}}=N$. The probability distribution of obtaining the variable $N_{\tilde{o}}$ is a binomial distribution of parameters $p=\mathcal{N}_{\tilde{o}} / \mathcal{N}$ and $N$,

$$
N_{\tilde{o}} \sim \operatorname{Bi}(N, p) .
$$

Using Eq. (A.7) and $\mathcal{N}_{\tilde{o}}+\mathcal{N}_{\sim \tilde{o}}=\mathcal{N}$, we obtain

$$
\mathcal{N}_{\tilde{o}}=\mathcal{L}^{2} / 2+\mathcal{L}^{2} / 2^{2 n_{o}}\left(\begin{array}{c}
2 n_{o}-1 \\
n_{o}
\end{array}\right) .
$$

Then, the probability $p$ is

$$
p=1 / 2+1 / 2^{2 n_{o}}\left(\begin{array}{c}
2 n_{o}-1 \\
n_{o}
\end{array}\right)
$$

in the case of even appearance of the state, and $p=1 / 2$ in case of odd appearance. 
We can obtain an expression for the expected value of $\sigma^{2}$ for a fixed side $\tilde{o}$,

$$
\sigma^{2}=<\left(N_{\tilde{o}}-N / 2\right)^{2}>
$$

Previous calculations allow us to compute the expected values in odd $\sigma_{o}^{2}=$ $<\left(N_{\tilde{o}}-N / 2\right)^{2}>_{o}$ and in even $\left.\sigma_{e}^{2}=<\left(N_{\tilde{o}}-N / 2\right)^{2}\right\rangle_{e}$ occurrences of the present state $\mu_{p}$, in order to find a closed expression for (B.4). From Eq. (B.1), the expected value and variance of the variable $N_{\tilde{o}}$ are known. By replacing them in $\sigma^{2}=<\left(N_{\tilde{o}}-N / 2\right)^{2}>=\operatorname{Var}\left(N_{\tilde{o}}-N / 2\right)+\left(N_{\tilde{o}}-n / 2\right)^{2}$, we obtain

$$
\sigma^{2}=N\left(\left(p^{2}-p\right)(N-1)+N / 4\right)
$$

and, by replacing the value of $p$ given by (B.3) in (B.5), we obtain

$$
\sigma_{e}^{2}=N / 4+\frac{N}{4} \frac{1}{2^{\left(4 n_{o}\right)}}\left(\begin{array}{c}
2 n_{o} \\
n_{o}
\end{array}\right)^{2}-\frac{1}{2^{\left(4 n_{o}+2\right)}}\left(\begin{array}{c}
2 n_{o} \\
n_{o}
\end{array}\right)^{2}
$$

and $\sigma_{o}^{2}=1 / 4$. By discarding the third term in (B.6), and by assuming that $n_{0}=\mathcal{H} / 2$, which is valid in the case of $M G_{\text {rand }}^{\text {prior }}$, we obtain the same result as in the $M G_{\text {rand }}$ case (see [10]):

$$
\sigma^{2}=N / 4+\frac{N}{4 \mathcal{H} \pi}
$$

Finally, the results for the symmetric phase in both $M G_{\text {rand }}$ and $M G_{\text {rand }}^{\text {prior }}$ coincide. Indeed, numerical simulations of the $M G$ and $M G^{\text {prior }}$ show that this holds during all phases of both games, and the only difference appears in the dispersion of the reduced variance $\sigma^{2} / N$ in the first region of the symmetric phase, as we could see in Figure 1.

\section{Acknowledgments}

G. Acosta and I. Caridi are members of the CONICET, Argentina. This work has been partially supported by ANPCyT under grant BID PICT 2007910.

[1] D. Challet, Y. C. Zhang, Emergence of cooperation and organization in an evolutionary game, Physica A 246 (1997) 407.

[2] D. Challet, M. Marsili, Y. C. Zhang, Minority Games, Oxford University Press (2005). 
[3] Y. C. Zhang, Modeling mechanism with evolutionary games, Europhys. News 29 (1998) 51.

[4] D. Challet, Y. C. Zhang, On the minority game: analytical and numerical studies, Physica A 256 (1998) 514.

[5] R. Savit, R. Manuca, R. Riolo, Adaptive competition, market efficiency, and phasse transitions, Phys. Rev. Lett. 82 (1999) 2203.

[6] A. Cavagna, Irrelevance of memory in the minority game, Phys. Rev. E 59 (1999) R3783.

[7] D. Challet, M. Marsili, Relevance of memory in minority games, Phys Rev. E 62 (2000) 1862.

[8] K. H. Ho, W. C. Man, F. K. Chow, H. F. Chau, Memory Is Relevant In The Symmetric Phase Of The Minority Game, Phys. Rev. E 71 (2005) 066120 .

[9] R. Manuca, Y. Li, R, Riolo, R. Savit, The structure of adaptive competition in minority game, Physica A 282 (2000) 574.

[10] G. Acosta, I. Caridi, S. Guala, J. Marenco, The Full Strategy Minority Game, Physica A 391 (2012) 217.

[11] P. Jefferies, M. L. Hart, N. F. Johnson, Deterministic dynamics in the minority game, Phys Rev. E 65 (2001) 016105.

[12] D. Zheng, B.H. Wang, Statistical properties of the attendance time series in the minority game, Physica A 301 (2001) 560.

[13] SS. Liaw, C.Liu, The quasi-periodic time sequence of the population in minority game, Physica A 351 (2005) 571.

[14] I. Caridi, H. Ceva, Minority game: a mean-field-like approach, Physica A 317 (2003) 247.

[15] G. Csardi, T. Nepusz, The igraph software package for complex network research, InterJournal, Complex Systems (2006) 1695 (http://igraph.sf.net). 
[16] R Development Core Team, R: A language and environment for statistical computing. R Foundation for Statistical Computing, Vienna, Austria. ISBN 3-900051-07-0 (2008), URL http://www.R-project.org.

[17] W.T. Tutte, Graph Theory, Cambridge University Press, 2001. 